\title{
Study of Printing Errors in Digitally Fabricated FSS
}

Badredin M.Turki, Edward A.Parker, M. Ali Ziai and John C. Batchelor, Veronica Sanchez-Romaguera and Stephen G. Yeates

This is an accepted pre-published version of this paper.

(C) 2013 IEEE. Personal use of this material is permitted. Permission from IEEE must be obtained for all other uses, in any current or future media, including reprinting/republishing this material for advertising or promotional purposes, creating new collective works, for resale or redistribution to servers or lists, or reuse of any copyrighted component of this work in other works.

The link to this paper is http://www.lapconf.co.uk/ 


\section{Study of Printing Errors in Digitally Fabricated FSS}

\author{
Badredin M.Turki, Edward A.Parker, M. Ali Ziai and \\ John C. Batchelor, \\ School of Engineering \\ University of Kent, Canterbury, UK \\ bmmt2@kent.ac.uk, e.a.parker@kent.ac.uk, \\ maz5@kent.ac.uk,j.c.batchelor@kent.ac.uk
}

\author{
Veronica Sanchez-Romaguera and \\ Stephen G. Yeates \\ Organic Materials Innovation Centre (OMIC), School of \\ Chemistry, University of Manchester, UK \\ Veronica.Sanchez@manchester.ac.uk, \\ Stephen.Yeates@manchester.ac.uk
}

\begin{abstract}
Low cost fabrication techniques might give rise to defects in electromagnetic structures. This paper investigates the detrimental effect of producing frequency selective screens with differing percentages of absent square loop elements. It is shown that as many as one in five elements can be incomplete before the array transmission response is considered unusable.
\end{abstract}

Keywords; Frequency selective surfaces; Indoor radio propagation; Inkjet printing.

\section{INTRODUCTION}

Inkjet technology is considered to be a promising means for economically manufacturing electromagnetic structures on inexpensive substrates such as paper, with a process that reduces the waste associated with chemical etching [1]. Inkjet drop-on-demand (DoD) technology [2] can deposit precise and repeatable drop sizes achieving the resolutions required for UHF printed circuits. Frequency Selective Surfaces (FSS) are well-known periodic structures with applications in reflector antennas, radome design, and satellite communications. They are filters of electromagnetic waves and printed FSS have been shown to be capable of achieving transmission nulls (signal attenuation) comparable in depth with those of conventionally etched bulk copper [3].

Particularly in the built environment, wireless communication system performance is limited by factors such as co-channel interference as the radio spectrum is heavily utilized in certain bands. Also user privacy may be compromised by eavesdropping with close-by receivers. These problems are pronounced in indoor wireless systems owing to the concentration of users sharing a limited number of unlicensed bands. The introduction of FSS into the building structure has been considered as a possible way to improve the situation [4],[5],[6].

In printed mass produced FSS, such as might be required in application to the built environment for electromagnetic architecture modification, imperfections might be expected from blockage of printer nozzles, poor surface quality, and non-uniformity in any conducting ink sintering process that might be adopted. To assess the consequences of this effect, some results from a study of the impact on transmission properties arising from randomly missing elements in a simple square loop FSS are presented here. Arrays of square loop elements [7] were used as demonstrators, as they are a commonly encountered FSS configuration. Square loop arrays can provide a common reflection band of about $25 \%$ over incident angles ranging from 0 to $50^{\circ}$. This paper provides some insight into how the reflection band is affected by the loss of elements.

\section{DESIGN METHODOLOGY}

The complete arrays contained 1080 patch square loops arranged in a square lattice format with length $L$ and periodicity $P$ equal to 6 and $7 \mathrm{~mm}$ respectively, as shown in Fig.1. The element width $w$ was $0.4 \mathrm{~mm}$ and conductor thickness was $0.01 \mathrm{~mm}$.

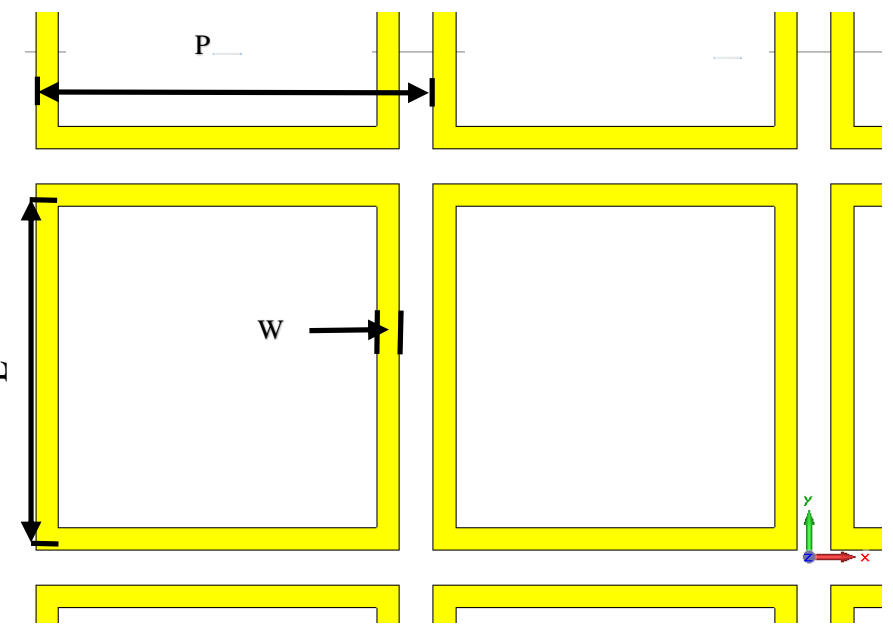

Fig.1 Finite square loop FSS

The FSS was etched onto a $0.045 \mathrm{~mm}$ thick polyester substrate with relative permittivity $\epsilon_{r}=3.5$ and loss tangent $=0.02$. The physical size of the array was $280 \times 190 \mathrm{~mm}^{2}$.

Four additional FSS were fabricated, with 10, 20, 30 and 40\% of the elements missing from random positions in the lattice. The numbers of the absent elements were 108, 216, 324, and 432 respectively. All arrays were placed in an aperture in an absorbing screen $1.18 \mathrm{~m}$ away from the illuminating horn antennas. 
The five arrays were also simulated using CST Microwave Studio $^{\text {TM }}$ (CST MWS) for comparison with measurement. Figs. 2 (a) \& (b) show the modelled complete array and also that with $40 \%$ absent elements.

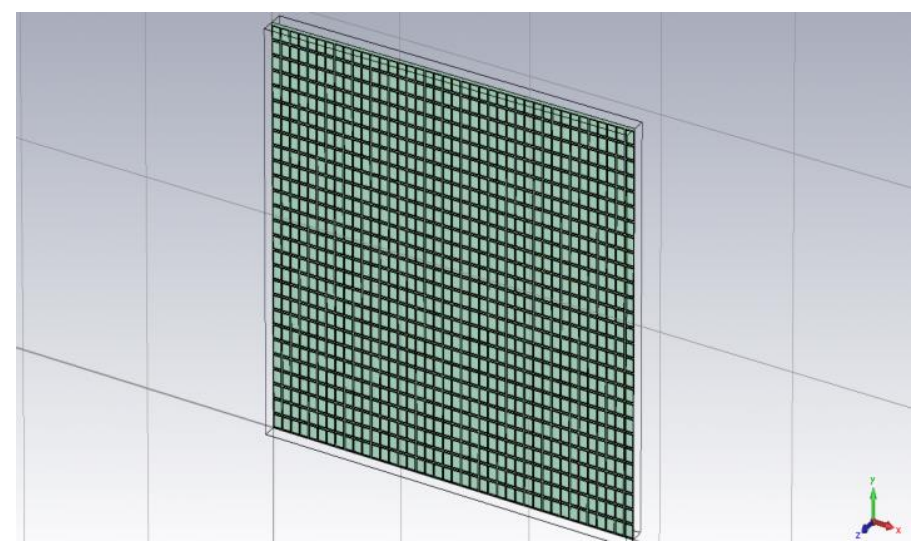

(a) Complete Screen

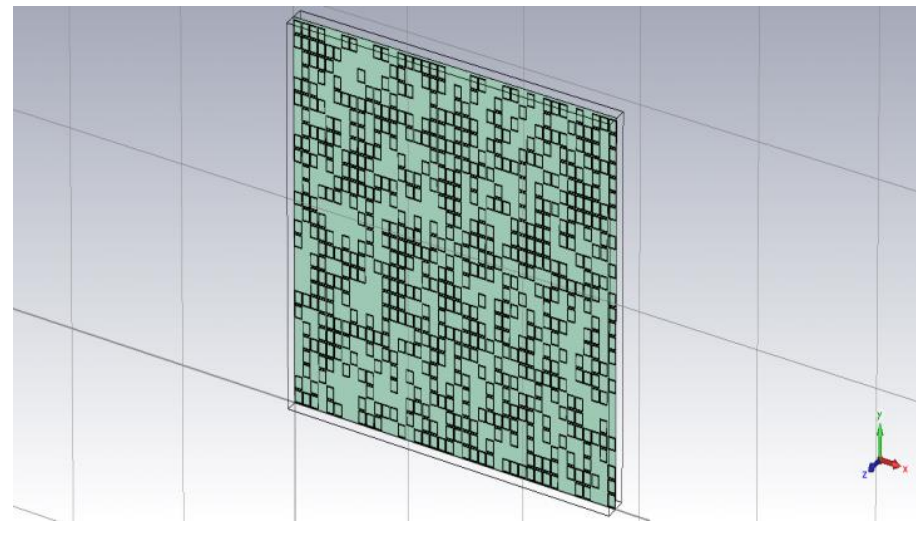

(b) Screen with $40 \%$ absent elements

Fig.2 Modelled Square loop FSS arrays

\section{RESULTS}

\section{A. Transmission response $\left(S_{21}\right)$}

The measured and simulated transmission responses $\left(\mathrm{S}_{21}\right)$ are shown in Fig.3(a) and Fig.3(b). As the number of missing elements increases, the transmission null degrades.

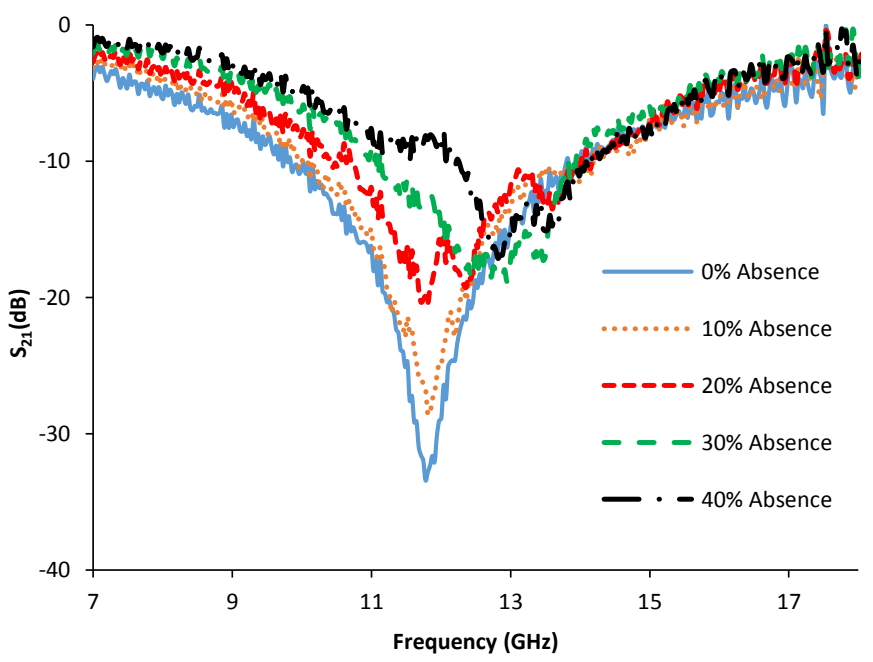

(a) Measured results

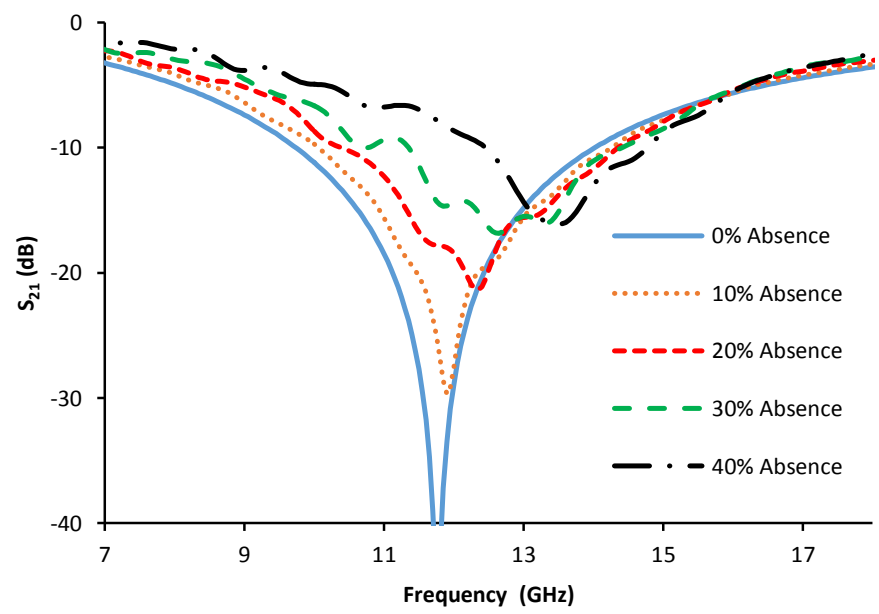

(b) Simulated results

Fig.3 Transmission response $\left(\mathrm{S}_{21}\right)$

The absence of $10 \%$ and $20 \%$ of the elements causes a reduction of about $6 \mathrm{~dB}$ and $16 \mathrm{~dB}$, respectively, in the measured transmission null depths. The depths in the simulated cases are broadly similar. Fig.4 summarises the results.

There is also a small shift in the resonant frequency which becomes more pronounced as the number of missing elements increases, as illustrated in Fig. 5. 


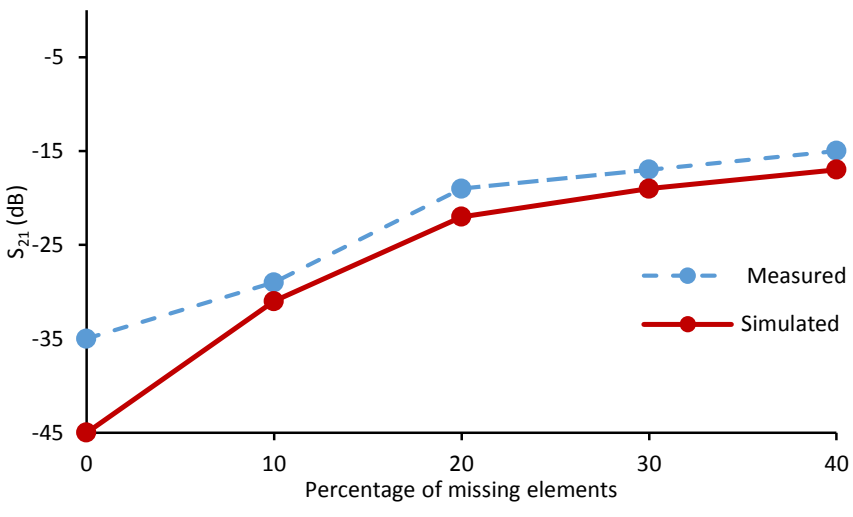

Fig.4 Depth of transmission null $\left(\mathrm{S}_{21}\right)$

The resonance frequency $f_{r}$ increases by about $300 \mathrm{MHz}$ when $10 \%$ of the elements are absent, while $20 \%$ absent elements lead to a $450-500 \mathrm{MHz}$ increase. As then might be deduced, a large increase, of about $1 \mathrm{GHz}$, occurs when 30 and $40 \%$ of the elements are absent.

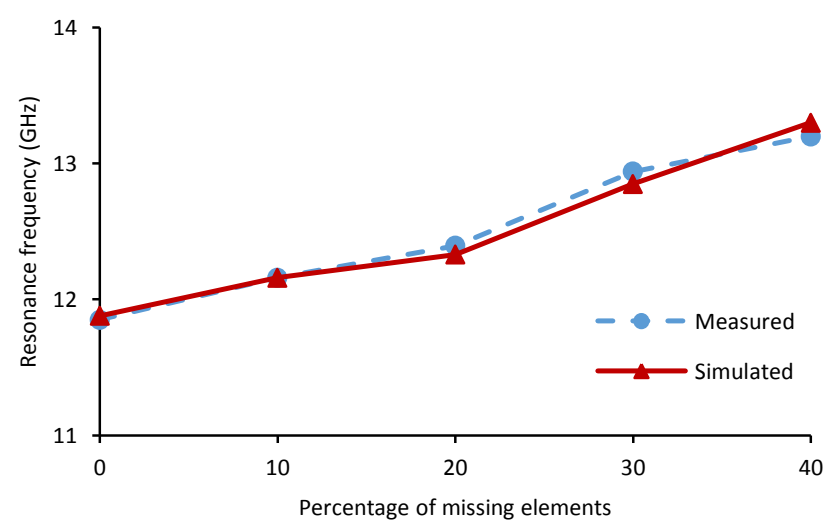

Fig.5 Resonance frequency

\section{B. $10 \mathrm{~dB}$ Reflection Bandwidth}

In addition to frequency drift, the available reflection bandwidth is also affected by element absence. The measured $-10 \mathrm{~dB}$ width for the complete FSS is about $4 \mathrm{GHz}$ and this is reduced by about $2 \%(50 \mathrm{MHz})$ and $20 \%(900 \mathrm{MHz})$, respectively for 10 and $20 \%$ missing elements (Fig.6). Larger bandwidth reductions of $30 \%$ and $70 \%$ are experienced when 30 and $40 \%$ of the elements are missing, a situation expected to be out of specification for any system.

The measured and simulated resonant frequencies, transmission null depths, and $10 \mathrm{~dB}$ bandwidth of the five square loop arrays are summarized in Table 1.

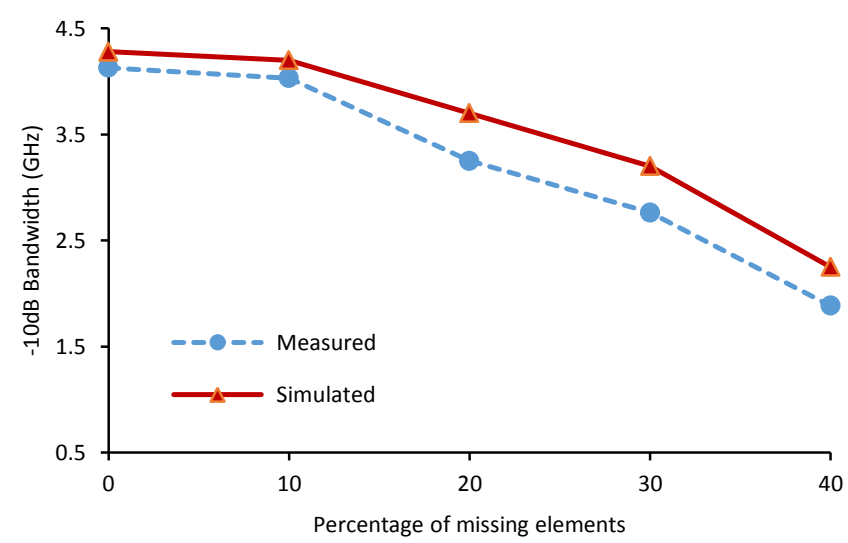

Fig.6 Reflection Bandwidth.

TABLE 1 SUMMARY OF RESULTS

\begin{tabular}{|c|c|c|c|c|c|c|}
\hline \multirow{2}{*}{$\begin{array}{c}\text { \% Absent } \\
\text { elements }\end{array}$} & \multicolumn{2}{|c|}{$\boldsymbol{f}_{\boldsymbol{r}}(\mathrm{GHz})$} & \multicolumn{2}{c|}{$\mathrm{S}_{21}(\mathrm{~dB})$} & \multicolumn{2}{c|}{$\mathrm{BW}(\mathrm{GHz})$} \\
\cline { 2 - 7 } & $\mathbf{S}$ & $\mathbf{M}$ & $\mathbf{S}$ & $\mathbf{M}$ & $\mathbf{S}$ & $\mathbf{M}$ \\
\hline 0 & 11.9 & 11.9 & -45 & -35 & 4.3 & 4.1 \\
\hline 10 & 12.2 & 12.2 & -31 & -29 & 4.2 & 4.0 \\
\hline 20 & 12.3 & 12.3 & -22 & -19 & 3.7 & 3.3 \\
\hline 30 & 12.8 & 12.9 & -19 & -17 & 3.2 & 2.8 \\
\hline 40 & 13.3 & 13.2 & -17 & -15 & 2.3 & 1.2 \\
\hline
\end{tabular}

\section{DISCUSSION AND CONCLUSIONS}

In a parallel study on the influence of defects in arrays of linear dipoles on squared and skewed lattices [8] it was again found that errors in $15-20 \%$ of the elements at random locations could be tolerated if a null depth of $20 \mathrm{~dB}$ was taken as a benchmark, although those FSS were slightly more sensitive to faults.

As an indication of the improvement that might be obtained through the use of FSS in buildings, suppressing the external interference by $15 \mathrm{~dB}$ can reduce the outage probability in mobile communications by more than a factor of 20 [9]. Furthermore, in square law propagation conditions a $10 \mathrm{~dB}$ attenuation of the co-channel interference level shortens the co-channel separation required, and therefore the cell size, by a factor of about three. The results presented here demonstrate that meeting such performance requirements should be readily achievable using manufacturing methods that do not meet the quality standards demanded for applications such as, for example, might be found in multiband satellite communication systems.

\section{ACKNOWLEDGMENT}

The authors wish to acknowledge the contribution of the UK EPSRC in funding this research through grant EP/J000086/1. 


\section{REFERENCES}

[1] G. Shaker, A. Rida, S. Safavi-Naeini, M. M. Tentzeris, and S. Nikolaou, "Inkjet printing of UWB antennas on paper based substrates," Antennas and Wireless Propagation Letters, IEEE, vol. 10, no. 2011, pp. 111.

[2] M. E. Solutions, "Digital Inkjet Printing for Etching Circuits," Journal of HKPCA, no. 30, pp. 16-21, 2008.

[3] J. C. Batchelor, E. A. Parker, J. A. Miller, V. SanchezRomaguera, and S. G. Yeates, "Inkjet printing of frequency selective surfaces," Electronics Letters, vol. 45, no. 1, p. 7, 2009.

[4] M. Hook \& Colleagues, "Project to demonstrate the ability of Frequency Selective Surface Structures to enhance the spectral efficiency of radio systems when used within buildings," Ofcom ref AY4464A, pp. 158, 2003.

[5] G. Sung, K. Sowerby, M. Neve, and A. Williamson, "A Frequency-Selective Wall for Interference Reduction in Wireless Indoor Environments," IEEE
Antennas and Propagation Magazine, vol. 48, no. 5, pp. 29-37, Oct. 2006.

[6] E. A. Parker, J. C. Batchelor, R. Chiang, A. G. Williamson, B. Sanz-Izquierdo, M. J. Neve, and K. W. Sowerby, "Frequency selectively screened office incorporating convoluted FSS window," Electronics Letters, vol.46, no.5, pp. 317-318, 2010.

[7] R. J. Langley and E. A. Parker, "Equivalent circuit model for arrays of square loops," Electronics Letters, vol. 18, no. 7, p. 294, 1982.

[8] B. M. Turki, E. A. Parker, M. A. Ziai, and J. C. Batchelor, "Influence of defective elements on the performance of Frequency Selective Surfaces," unpublished.

[9] A. H. Wong, M. J. Neve, and K. W. Sowerby, "Performance analysis for indoor wireless systems employing directional antennas in the presence of external interference," 2005 IEEE Antennas and Propagation Society International Symposium, Washington, DC, USA, vol. 1A, pp. 799-802. 